Commun. math. Phys. 2, 384-397 (1966)

\title{
On some Groups of Automorphisms of Physical Observables
}

\author{
G. F. Dell'Antonio* \\ Institut des Hautes Etudes Scientifiques Bures-sur-Yvette \\ and \\ Courant Institute of Mathematical Sciences New York
}

Received December 20, 1965

\begin{abstract}
Given a weakly continuous one-parameter group of automorphisms of a $C^{*}$-algebra $\mathfrak{A}$ of operators on a Hilbert space we show that it is implementable by a strongly continuous one-parameter group of unitary operators belonging to the weak closure of $\mathfrak{A}$, provided that a certain condition - akin to the boundedness from below of the spectrum of the generators - is satisfied.
\end{abstract}

In recent years, some results have been obtained on special oneparameter groups of automorphisms of a set of observables.

Consider, in particular, the case in which the observables are represented in a Hilbert space $\mathscr{H}$ by the weak closure $\mathfrak{A}^{-}$of the union of algebras associated with bounded space-time regions. Let the group of translations in the direction $a_{\mu}$ be induced by a strongly continuous oneparameter group of unitary operators on $\mathscr{H}$, with generator $T_{a_{\mu}}$. In this framework, ARAkI [1] has shown that if $T_{a_{\mu}}$ has spectrum bounded below and if the lowest point in the spectrum corresponds to at least one eigenvector $\xi \in \mathscr{H}$, then $T_{a_{\mu}}$ is affiliated to $\mathfrak{A}^{-}$(in the sense that each projection of $T_{a_{\mu}}$ belongs to $\left.\mathfrak{A}^{-}\right)$.

When the generator $T$ is bounded, the infinitesimal form of a group of automorphisms is a derivation of $\mathscr{Q}$ - induced by $T^{\mathbf{1}}$.

Recently, KADISON [2] has proved the following remarkable theorem:

Theorem $\alpha$. Let $\mathfrak{A}$ be a $C^{*}$-algebra ${ }^{2}$ of operators on a Hilbert space $\mathscr{H}$ and let $\delta: A \rightarrow \delta(A)$ be a derivation of $\mathfrak{Q}$. Then there exists a bounded operator $T$ in $\mathfrak{A}^{-}$(the weak closure of $\mathfrak{Q}$ ) such that $\delta(A)=[A, T]$ for all $A \in \mathfrak{A}$.

An immediate consequence is

* On leave from the Istituto di Fisica Teorica, Universitá di Napoli.

1 A derivation of an algebra $\mathfrak{A}$ is a linear map $\delta$ from $\mathfrak{A}$ to $\mathfrak{A}$ such that $\delta(A B)$ $=\delta(A) \cdot B+A \cdot \delta(B)$ for all $A, B \in \mathfrak{A}$. If $\mathfrak{A}$ is an algebra of operators on a Hilbert space $\mathscr{H}$ and $T$ is a bounded operator, and if $[A, T] \in \mathfrak{U}$ for all $A \in \mathfrak{A}$, then the map $\delta_{T}$ defined by $\delta_{T}(A) \equiv[A, T]$ is a derivation of $\mathfrak{A}$, which is said to be induced by $T$.

2 For definitions and some results on $C^{*}$-algebras, see e.g., Dixmrer, les $C^{*}$. algebres et leur representations, Gauthier-Villars, Paris, 1964. 
Corollary $\alpha$. Let $\tau_{t}$ be a norm-continuous group of automorphisms of $\mathfrak{A}$, with bounded infinitesimal generator $T^{3}$. Then $T$ can be chosen to belong to $\mathfrak{A}^{-}$.

The purpose of this note is to extend Corollary $\alpha$ to certain weakly continuous groups of automorphisms of the algebra $\mathfrak{A}$. We shall not make explicit assumptions about the algebra - (e.g. whether it has or not a "local" structure) but will introduce two assumptions on the automorphisms. One of them is akin to the spectrum condition. The other guarantees that each automorphism in the group can be extended to the weak closure.

Let $\mathfrak{A}$ be a $C^{*}$-algebra of operators on a Hilbert space $\mathscr{H}$. Let $\tau_{t}$ be a one-parameter family of automorphisms of $\mathfrak{A}$, weakly continuous in the sense that ${ }^{4}$

$$
\left\langle\varphi, \tau_{t}(A) \psi\right\rangle
$$

is a continuous function of $t$ for every $\varphi, \psi \in \mathscr{H}$ and $A \in \mathfrak{A}$.

Consider in $\mathfrak{A}$ the left ideal $\mathfrak{I}[3]$ generated by elements $B \in \mathfrak{A}$ of the form

$$
\begin{aligned}
B=\int A(t) f(t) d t, A \in \mathcal{A}, A(t) & =\tau_{t}(A), f \in L_{1}{ }^{5}, \\
\operatorname{supp} f \cap\{p: p>-M\} & =0,
\end{aligned}
$$

for some fixed $M>0^{6}$.

Let $\mathscr{K}$ be the set of vectors $\varphi \in \mathscr{H}$ such that $\mathfrak{\Im} \varphi=0$. This set is linear, and closed in the Hilbert space topology since it is the intersection of linear closed spaces, the null spaces of the elements of $\mathfrak{I}$. There exists therefore a projection $\pi$ such that $\mathscr{K}=\pi \mathscr{H}$, and we have $\Im \pi=0$. Our first assumption is

Postulate $\alpha .{ }^{7} \pi$ is not the null operator and its central support is $1 .{ }^{8}$ We shall note, for future use, that $\tau(\mathfrak{J})=\mathfrak{J}$.

${ }^{3} T$ is of course only defined modulo addition of elements in $\mathfrak{A}^{\prime}$, the commutant of $\mathfrak{A}$.

${ }^{4}$ We use the notation $\langle\varphi, \psi\rangle$ for the scalar product in $\mathscr{H} ;\langle\varphi, \psi\rangle$ is taken linear in $\psi$ and anti-linear in $\varphi$.

${ }^{5} L_{1}$ is the set of functions of such that $\int|f(t)| d t<\infty$.

6 The choice of $M$ is irrelevant, insofar as $M>0$. It will be evident in what follows that Postulates $\alpha$ and $\beta$ will be satisfied for all $M>0$ if they are satisfied for some $M_{0}>0$. If, however, Postulate $\alpha$ is satisfied with $M=0$, then Postulate $\beta$ is unnecessary and Theorem 1 and Propositions 1 to 5 are trivial. In this case there exists at least one eigenstate of $H$ (as defined in Theorem 2) to the eigenvalue zero.

7 If the automorphisms $\tau_{b}$ were induced by a strongly continuous one-parameter group of unitary operators on $\mathscr{H}$ with generator $H$, Postulate $\alpha$ would be satisfied if $H$ were bounded below (in each superselection sector, if there are more than one). For this reason we shall refer to Postulate $\alpha$ as "spectrum condition".

${ }^{8}$ Postulate $\alpha$ is independent in the sense that one can construct examples in which every other condition (and also $\pi \neq 0$ ) is met but the continuous group of unitary operators inducing $\tau_{t}$ cannot be chosen in $\mathfrak{A}^{-}$. 


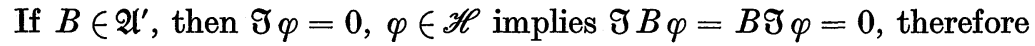
$\pi B \pi=B \pi$. Taking adjoints with $B$ self-adjoint (if $B \in \mathfrak{A}^{\prime}, B+B^{*}$ and $i\left(B-B^{*}\right)$ both belong to $\left.\mathfrak{A}^{\prime}\right) \pi B \pi=\pi B$. Therefore $B \pi=\pi B$, i.e. $\pi \in \mathfrak{Q}^{-}$.

Consider now the set $\mathfrak{A}_{\pi} \stackrel{\text { def }}{=} \pi \mathfrak{A} \pi$. This set is not an algebra in general, since it is not closed under multiplication (but is an algebra if $\mathfrak{U}$ is weakly closed). For each $\tau_{t}$ on $\mathfrak{A}$ we would like to define an automorphism $\tau_{t}$ on some $C^{*}$-algebra $\mathfrak{B}$ satisfying $\mathfrak{A}_{\pi} \subset \mathfrak{B} \subset \mathfrak{A}_{\pi}^{-}$. If $\mathfrak{A}$ is weakly closed, this is done by choosing for $\tau_{\pi}^{t}$ the restriction of $\tau_{t}$ to $\mathfrak{A}_{\pi}$. If $\mathfrak{A}$ is not weakly closed, we have not succeeded in proving that this can always be done except if one can choose $M=0$ in the definition of the ideal I. See footnote (9). To go further, we shall introduce, therefore,

Postulate $\beta .{ }^{10}$ Each automorphism $\tau_{t}$ of $\mathfrak{A}$ can be "extended" to an automorphism $\hat{\tau}_{t}$ of $\mathfrak{H}_{\pi}^{-}$such that $\hat{\tau}_{t}(\pi A \pi)=\pi \tau_{t}(A) \pi$ for all $A \in \mathfrak{A} . \tau_{t}$ need not be a priori weakly continuous in $t$.

A detailed analysis of Postulate $\beta$ and necessary and sufficient conditions for its validity have been given in [4] (Remark 2.2.3) in terms of permanent null sets. We shall only recall ${ }^{10}$ that, in the notations used so far, the isomorphic map $\tau_{t}$ extends to a weakly continuous isomorphism $\hat{\tau}_{t}$ of $\mathfrak{A}-$ if and only if for each $\xi \in \mathscr{H}$ the state $\varrho_{\xi}$ of $\mathfrak{A}$ defined by

$$
\varrho_{\xi}(A)=\left\langle\xi, \tau_{t}(A) \xi\right\rangle
$$

is weakly continuous on the unit sphere in $\mathfrak{A}$ at 0 , and has therefore a weakly continuous extension to the unit sphere in $\mathfrak{A}^{-} .{ }^{11}$

In other words, the mapping $T$ on the states of $\mathfrak{A}$ defined by

$$
T \cdot \varrho(A)=\varrho(\tau(A))
$$

must map vector states into normal states, and in fact the normal states onto themselves. In particular, if $\mathfrak{A}$ acts irreducibly on $\mathscr{H}, T$ must map the vector states onto themselves. In this case it is known ${ }^{12}$ that $\tau$ is implemented by a unitary transformation.

We shall now proceed in our analysis.

Notice first that for each $t, \hat{\tau}_{t}(\pi)=\pi$. In fact, from $\tau(\Im)=\Im$, one has

$$
0=\Im \pi=\hat{\tau}_{t}(\Im \pi)=\Im \hat{\tau}_{t}(\pi) .
$$

$\pi$ is uniquely characterized by $\Im \pi=0$; therefore $\hat{\tau}_{t}(\pi)=\pi$. q.e.d. We can therefore define, for each $t$, a mapping $\tau_{t}^{\pi}$ of $\mathfrak{Q}_{\pi}^{-13}$ onto itself as the

- If $\mathfrak{A}$ is not weakly closed and the spectrum condition is not satisfied, we can give examples in which the conditions of Postulate $\beta$ are not satisfied.

10 Ref. [4], Remark 2.2.3 and Coroll. 2.3.

11 A normal state of a (concrete) $C^{*}$-algebra $\mathfrak{A}$ is a state which is weakly continuous on the unit sphere of $\mathfrak{A}-$. A state $\varrho$ is called vector if there exists a vector $\xi \in \mathscr{H}$ such that $\varrho(A)=\langle\xi, A \xi\rangle$ for all $A \in \mathcal{A}$.

12 Ref. [4], Lemma 4.1.6.

13 Since $\pi \in \mathfrak{A}^{-},\left(\mathfrak{A}_{\pi}\right)^{-}=\mathfrak{A}_{\pi}^{-} \equiv \pi \mathfrak{A}^{-} \pi$. See e.g. Ref. [6], p. 18, Prop. 1 . 
restriction of $\hat{\tau}_{t}$ to $\mathfrak{Q}_{\pi}^{-}$. Notice also that $\tau_{t}^{\pi}(\pi A \pi)=\pi \tau_{t}(A) \pi$ for all $A \in \mathfrak{A}^{-}$. We shall now prove

Theorem 1. Let $\mathfrak{A}, \pi, \tau_{t}$ be defined as above. Then there exists a $C^{*}$-algebra $\mathfrak{B} \subset \mathfrak{A}_{\pi}^{-}$such that the mapping $\delta$ defined by

is a derivation in $\mathfrak{B}$.

$$
A \stackrel{\delta}{\longrightarrow} s-\lim _{t \rightarrow 0} \frac{A(t)-A}{t}
$$

We notice first that, for every pair $\varphi, \psi \in \mathscr{H}_{\pi}$ (not necessarily normalized), and $A \in \mathfrak{A}_{\pi}$, the function $f(t) \equiv\langle\varphi, A(t) \psi\rangle$ is entire of exponential type $M^{14}$. In fact, $f(t)$ is bounded (by $\|A\|\|\varphi\|\|\psi\|$ ), continuous and, by the definition of $\mathscr{H}_{\pi}$, such that

$$
\int f(t) g(t) d t=0
$$

for any $g \in \mathscr{L}_{\mathbf{1}}$ such that supp $\tilde{g} \cap\{p:-M \leqq p \leqq M\}=0$.

Therefore $f(p)$ has support in $\{p:-M \leqq p \leqq M\}$ and, by the PaleyWiener theorem [5], its Fourier transform $\tilde{f}(t)=f(t)$ is entire of exponential type $M$.

To simplify the discussion, let us introduce the set $\mathfrak{M}$ defined as follows:

$\mathfrak{M} \stackrel{\text { def }}{=}\left\{A: A \in \mathfrak{A}_{\pi}^{-},\|A(t)\|=\|A\| \forall t,\langle\varphi, A(t) \psi\rangle\right.$

We want to prove entire of exponential type $M$ for all $\left.\varphi, \psi \in \mathscr{H}_{\pi}\right\}$.

Proposition 1. The linear set $\mathfrak{M}$ is closed under multiplication and under the operation of taking uniform limits and adjoints. In other words, $\mathfrak{M}$ is a (concrete) $C^{*}$-algebra of operators.

That $\mathfrak{M}$ is closed under the operation of taking adjoints can be verified trivially.

a) Let $A, B \in \mathfrak{M}$. Then, for every pair $\varphi, \psi \in \mathscr{H}_{\pi}$, the function $\langle\varphi, A(t) B(t) \psi\rangle$ is entire of exponential type $2 M$.

We shall prove in Appendix 1, for every $\varphi, \psi \in \mathscr{H}_{\pi}$, there exist a sequence $A_{1}, A_{2}, \ldots, A_{n} \in \mathfrak{M} \forall n$, such that

$$
\left\langle\varphi, A_{n}(t) \psi\right\rangle \underset{n \rightarrow \infty}{\longrightarrow}\langle\varphi,(A B)(t) \varphi\rangle
$$

when $t \in I_{0}$, where $I_{0}$ is some denumerably infinite set of points which we choose dense in the real $t$-axis.

Since all the functions involved are entire of finite exponential type, this implies convergence on the whole $t$-axis.

But then (Appendix 2) $\langle\varphi,(A B)(t) \psi\rangle$ is entire of exponential type $M$.

Also,

$$
\left\|A B\left(t_{1}\right)\right\|=\lim _{n \rightarrow \infty}\left\|A_{n}\left(t_{1}\right)\right\|=\left\|A B\left(t_{2}\right)\right\| \quad \text { for any } t_{1}, t_{2} \in I_{0} .
$$

${ }^{14}$ This is a short-hand notation for: $f(t)$ is the restriction to the real axis of a function $f(z)$ entire and of exponential type $M$. 
A continuous function of $t$, constant on a dense set $I_{0}$, is constant everywhere. Therefore $A B \in \mathfrak{R}$. q.e.d.

b) Let $A_{n}(t) \underset{n \rightarrow \infty}{\longrightarrow} A(t)$ (uniform convergence), $A_{n} \in \mathfrak{M}, n=1,2, \ldots$ Using the fact that $\left\|A_{n}(t)-A_{m}(t)\right\|$ is independet of $t$, we obtain

$$
\begin{aligned}
&|\langle\varphi,(A(t+s))-A(s) \psi\rangle| \leqq \mid\left\langle\varphi,\left(A(t+s)-A_{n}(t+s)\right) \varphi\right|+ \\
&+\left|\left\langle\varphi\left(A(s)-A_{n}(s)\right) \psi\right\rangle\right|+\left|\left\langle\varphi\left(A_{n}(t+s)-A_{n}(s)\right) \psi\right\rangle\right| \leqq \\
& \leqq \mathbf{1} / \mathbf{3}+\mathbf{1} / \mathbf{3}+\mathbf{1} / \mathbf{3}=\mathbf{1}
\end{aligned}
$$

if $t$ is small enough.

Therefore for any $\varphi, \psi \in \mathscr{H},\langle\varphi, A(t) \psi\rangle$ is a continuous function and moreover, there exists an integer $n_{0}$ such that

$$
\left|\langle\varphi, A(t) \psi\rangle-\left\langle\varphi, A_{n}(t) \psi\right\rangle\right|<1 \quad \text { if } n>n_{0} \text {, independently of } \mathrm{t} \text {. }
$$

From the results of Appendix 2, $\langle\varphi, A(t) \psi\rangle$ is entire of exponential type $M$ : since in addition

$$
\|A(t)\|=\lim _{n \rightarrow \infty}\left\|A_{n}(t)\right\|=\lim _{n \rightarrow \infty}\left\|A_{n}\right\|=\|A\|
$$

one has finally $A \in \mathfrak{M}$. q.e.d.

The next step is to prove

Proposition 2. The relation $A(t) \stackrel{d}{\longrightarrow} s-\operatorname{limit}_{s \rightarrow 0} \frac{A(s+t)-A(t)}{s} \stackrel{\text { def }}{=} A^{\prime}(t)$ defines a map from $\mathfrak{W}$ to $\mathfrak{W}$.

Notice first that, since $\mathfrak{Q}_{\boldsymbol{\pi}}^{-}$is strongly closed, if $A^{\prime}(t)$ exists, it belongs to $\mathfrak{A}_{\pi}^{-}$for all $t$.

To prove the existence of $A^{\prime}(t)$ we shall show first that, for every real $t$ and $\varphi \in \mathscr{H}_{\pi}$, the sequence $\frac{A(s+t)-A(t)}{s} \varphi$ converges in the Hilbertspace norm when $s \rightarrow 0$. To simplify notations, let us consider explicitly the case $t=0$. We must prove

where

$$
\lim _{8, t \rightarrow 0} h(s, t)=0
$$

$$
h(s, t) \stackrel{\text { def }}{=}\left\langle\varphi,\left(\frac{A(t)-A}{t}-\frac{A(s)-A}{s}\right)^{*}\left(\frac{A(t)-A}{t}-\frac{A(s)-A}{s}\right) \varphi\right\rangle
$$

Without loss of generality we shall take $A$ self-adjoint; since $\tau_{t}$ is *-isomorphism, $A(t)$ is thenself-adjoint for all $t$.

With the notation $f(s, t) \stackrel{\text { def }}{=}\langle\varphi, A(s) A(t) \varphi\rangle$ we know that $f(p, q)$ has support contained in $-M \leqq p \leqq M,-M \leqq q \leqq M$. Therefore [5] there exists a function $f(\xi, \eta)$ entire in the direct product of the $\xi, \eta$ planes and coinciding with $f(s, t)$ on the real plane. In particular, 
$f(s, t)$ can be expanded in a convergent power series in $s, t$ around the origin. An easy substitution shows then that

$$
h(s, t)=\left.\frac{\partial^{4} f}{\partial s^{2} \partial t^{2}}\right|_{s=t=0} \cdot(s-t)^{2}+\text { higher order terms . }
$$

One can then find $\delta>0$ such that, for $|t|<\delta,|h(s, t)|<1$. q.e.d.

The operator $A^{\prime}(t)$ we have defined is, for every $t$, linear, homogeneous and also symmetric, since, for $\varphi, \psi \in D_{A(t)}$

$$
\begin{aligned}
\left\langle\varphi, A^{\prime}(t) \psi\right\rangle & =\lim _{s \rightarrow 0}\left\langle\varphi, \frac{A(t+s)-A(t)}{s} \psi\right\rangle \\
& =\lim _{t \rightarrow 0}\left(\left\langle\psi, \frac{A(t+s)-A(t)}{s} \varphi\right\rangle\right)^{*}=\left\langle\varphi, A^{\prime}(t) \psi\right\rangle^{*} .
\end{aligned}
$$

Hence $A^{\prime}(t)$ is, for every $t$, a linear homogeneous symmetric operator defined everywhere and therefore [6] (Ch. VIII, No. 114) by the HellingerToeplitz theorem, a bounded operator.

To complete the proof of Proposition 2, we have to show that $\left\langle\varphi, A^{\prime}(t) \psi\right\rangle$ is an entire function of exponential type $M$ for all $\varphi, \psi \in \mathscr{H}_{\pi}$ and also that $\left\|A^{\prime}(t)\right\|$ is independent of $t$.

The first assertion follows immediately from the fact that $\left\langle\varphi, A^{\prime}(t) \psi\right\rangle$ $=\frac{\partial}{\partial t}\langle\varphi, A(t) \psi\rangle$. As for the second, we recall that strong convergence implies convergence of the norms. Therefore

$$
\left\|A^{\prime}(t)\right\|=\lim _{s \rightarrow 0}\left\|\frac{A(t+s)-A(t)}{s}\right\|=\lim _{s \rightarrow 0} \frac{\|A(s)-A(0)\|}{s}=\left\|A^{\prime}(0)\right\|
$$

We next prove for all $t$. q.e.d.

Proposition 3. The map $d$ defined in Proposition 2 is a derivation of the $C^{*}$-algebra $\mathfrak{W}^{15}$.

It must be proved that, if $A, B \in \mathfrak{M},(A B)^{\prime}=A^{\prime} B+A B^{\prime}$. We already know that the limits which define $(A B)^{\prime}, A^{\prime}$ and $B^{\prime}$ exist in the strong (and therefore also weak) sense. We have only to check that

$$
\lim _{t \rightarrow 0} h_{\varphi}(t)=0 \quad \forall \varphi \in \mathscr{H}_{\pi}
$$

where setting $A=\left.A(t)\right|_{t=0}$ etc.,

$$
h_{\varphi}(t) \stackrel{\text { def }}{=}\left\langle\varphi, \frac{A(t) B(t)-A B}{t}-\frac{A(t)-A}{t} B-A \frac{B(t)-B}{t} \varphi\right\rangle .
$$

Let $f_{\varphi}(s, t) \stackrel{\text { def }}{=}\langle\varphi, A(s) B(t) \varphi\rangle$. The function $f_{\varphi}(s, t)$ is entire in the product of the $s, t$ planes and, for $t$ small enough,

$$
h_{\varphi}(t)=\left.\frac{\partial^{2} f}{\partial s \partial t}\right|_{s=t=0} \cdot t+0\left(t^{2}\right) . \quad \text { q.e.d. }
$$

15 The map $d$ is continuous in the uniform topology. This follows from the general theory of derivations of $C^{*}$-algebra [7]; in our particular case, it can be easily checked using BERNSTEIN's theorem [8] which states that, if $f(z)$ is entire of exp. type $M$ and if $|f(t)| \leqq 1$ for all real $t$, then $\left|f^{\prime}(t)\right| \leqq M$ for all real $t$. 
Theorem 1 follows now from Propositions $1-3$; we can take, e.g., for $\mathfrak{B}$ the $C^{*}$ algebra generated by the elements in $d^{n} \cdot \mathfrak{A}_{\pi}$ for every finite $n$. Obviously, $\mathfrak{B}^{-}=\mathfrak{A}_{\pi}^{-}$.

Combining Theorem $\alpha$ and Theorem 1, we obtain

Proposition 4. There exists a self-adjoint operator $H_{\pi} \in \mathfrak{A}_{\pi}^{-}$such that

$$
A^{\prime}=i\left[A, H_{\pi}\right] \text { for all } A \in \mathfrak{B} \text {. }
$$

That $H_{\pi}$ can be chosen self-adjoint follows because if $A$ is self adjoint $A^{\prime}$ is also; therefore $H_{\pi}, H_{\pi}^{*}$ and $\frac{1}{2}\left(H_{\pi}+H_{\pi}^{*}\right)$ generate the same derivation of $\mathfrak{B}$.

We can write then, for $\varphi, \psi \in \mathscr{H}_{\pi}$, with $\left.A(t)\right|_{t=0}=A$,

$$
\left\langle\varphi, A^{\prime} \psi\right\rangle=\frac{\partial}{\partial t}\langle\varphi, A(t) \psi\rangle_{\psi=0}=i\left\langle\varphi,\left[A, H_{\pi}\right] \psi\right\rangle .
$$

By iteration

$$
\frac{\partial^{n}}{\partial t^{n}}\langle\varphi, A(t) \psi\rangle_{t=0}=i^{n}\left\langle\varphi,\left[\left[[A, \underbrace{\left.\left.\left.H_{\pi}\right], H_{\pi}\right] \ldots H_{\pi}\right]}_{n \text { times }} .\right.\right.\right.
$$

Since $\langle\varphi, A(t) \psi\rangle$ is entire, we conclude

Proposition 5. For all $A \in \mathfrak{A}_{\pi}$ one has

$$
A(t+s)=e^{-i H_{\pi} t} A(s) e^{i H_{\pi} t}
$$

and this relation extends by continuity to $\mathfrak{A}_{\pi}^{-}$.

This means that the automorphisms $\tau_{t}^{\pi}$ of $\mathfrak{A}_{\pi}^{-}$are implemented by the unitary operators $U(t)=e^{i H_{\pi} t} \in \mathfrak{U}_{\pi}^{-}$for all $t$.

Our next task is to show that also the one-parameter group of automorphisms $\tau_{t}$ of $\mathfrak{A}$ is implemented by a strongly continuous oneparameter group of unitary operators $V_{t} \in \mathfrak{A}^{-}$with infinitesimal generator $H$.

We shall first prove the following lemma ${ }^{16}$.

Lemma 1. Let $\mathfrak{A}^{-}$be a Von-Neumann algebra ${ }^{17}, \pi$ a projection in $\mathfrak{A}^{-}$. Let $\psi$ be a vector in $\pi \mathscr{H}$ such that the set $\mathscr{N}_{\psi} \equiv\left\{\sum_{n} A_{n} A_{n}^{\prime} \psi\right\}$ is dense in $\mathscr{H}$.

Let $\tau$ be a $C^{*}$-automorphism of $\mathfrak{A}^{-}$. Suppose that there exists a unitary operator $U, U \in \mathfrak{A}_{\pi}^{-}$, such that $\tau(A)=U A U^{-1}$ for all $A \in \mathfrak{A}_{\pi}^{-}$. Then there exists a unitary operator $V \in \mathfrak{A}^{-}$such that $\tau(A)=V A V^{-1}$ for all $A \in \mathfrak{A}^{-}$, and $V=U$ on $\mathscr{H}_{\pi}$.

The proof will consist in the construction of the operator $V$. By assumption, the set $\mathfrak{T}_{\psi} \equiv\left\{\sum_{n} A_{n} A_{n}^{\prime} \psi\right\}$ is dense in $\mathscr{H}$ for some $\psi \in \pi \mathscr{H}$. All sums here are finite and $A_{n}, A_{n}^{\prime}$ are arbitrary elements in $\mathfrak{A}, \mathfrak{A}^{\prime}$ respectively.

16 The proof presented here has been suggested to me by H. J. Borchers. A similar proof is given in [9].

17 Weakly closed $C^{*}$-algebra of operators on a Hilbert space. 
We define on $\mathfrak{T}_{\psi}$ an operator $V$ by the following prescription:

$$
V \cdot \sum_{n} A_{n} A_{n}^{\prime} \psi=\sum \tau\left(A_{n}\right) \cdot A_{n}^{\prime} U \psi .
$$

This prescription defines a linear, homogeneous operator on $\mathcal{2}_{\psi}$ if we can show that $\varphi \in \mathfrak{R}_{\psi}$ and $V \varphi=0$ imply $\varphi=0$. If $\varphi \in \mathfrak{R}_{\psi}$ the norm of $V \varphi$ is

$$
\begin{aligned}
\|V \varphi\| & =\sum_{m, n}\left\langle\psi, U^{*} A_{n}^{\prime *} \pi \tau\left(A_{n}^{*}\right) \tau\left(A_{m}\right) \pi A_{m}^{\prime} U \psi\right\rangle \\
& =\sum_{m, n}\left\langle\psi, A_{n}^{\prime *} U^{*} \tau\left(\pi A_{n}^{*} A_{n} \pi\right) A_{m}^{\prime} U \psi\right\rangle=\|\varphi\| .
\end{aligned}
$$

We have used the fact that $\pi$ commutes with $U$ and $\mathfrak{Y}^{\prime}$, and the identities $\pi \psi=\psi$ and $\tau(\pi)=\pi$. We have also made use of the property that $\tau$, when restricted to $\mathfrak{A}_{\pi}^{-}$, is induced by $U$.

The identity (16) shows that $V$ is defined on $\mathfrak{T}_{\psi}$ and is uniformly bounded there, and also that $V^{+} V=1$ on $\mathfrak{P}_{\psi} . V$ can therefore be extended by continuity to $\mathscr{H}$ and $V^{+} V=1$ on $\mathscr{H}$. To prove unitarity, we shall show that the range of $V$ is dense in $\mathscr{H}$. Assume to the contrary that there exists a vector $\xi, \xi \in \mathscr{H},|\xi|=1$, such that $\langle\xi, V \varphi\rangle=0$ $\forall \varphi \in \mathscr{H},|\varphi|=1$.

Let $\varphi \in \mathscr{T}_{\psi}, \varphi=\sum A_{n} A_{n}^{\prime} \psi$. Then $\langle\xi, V \varphi\rangle=\sum_{n}\left\langle\xi, \tau\left(A_{n}\right) A_{n}^{\prime} U \psi\right\rangle=0$ for all choices of $A_{n}, A_{n}^{\prime}$. But $\tau(\mathfrak{A})=\mathfrak{A}$ and $\mathfrak{P}_{U \psi}$ is dense in $\mathscr{H}$. Therefore $\langle\xi, \varphi\rangle=0 \forall \varphi \in \mathfrak{R}_{U \psi} \Rightarrow \xi=0$, a contradiction.

To prove that $V \in \mathfrak{A}^{-}$, let $B$ be any element in $\mathfrak{A}^{\prime}$. For every $\varphi \in \mathfrak{A}_{\psi}$ we have

$$
V B \varphi=V \sum_{n} A_{n} B A_{n}^{\prime} \psi=\sum_{n} \tau\left(A_{n}\right) B A_{n}^{\prime} U \psi=B V \psi .
$$

Since $B$ is arbitrary in $\mathfrak{A}^{\prime}$ and $\mathfrak{T}_{\psi}$ is dense in $\mathscr{H}, V \in \mathfrak{A}^{-}$.

Also, $\pi V \pi=\pi U \pi$. In fact, again on $\mathfrak{R}_{\psi}$,

$$
\begin{aligned}
\pi V \pi \sum_{n} A_{n} A_{n}^{\prime} \psi=\pi \sum_{n} \tau\left(\pi A_{n} \pi\right) U A_{n}^{\prime} \psi & =\pi U \sum_{n} \pi A_{n} \pi A_{n}^{\prime} \psi \\
& =\pi U \pi \varphi
\end{aligned}
$$

and the identity extends to $\mathscr{H}$ by continuity.

We shall need one more identity, namely for $B \in \mathfrak{A}_{\pi}^{-}, \psi \in \mathscr{H}_{\pi}$,

$$
V \sum_{n} A_{n} A_{n}^{\prime} B \psi=\sum \tau\left(A_{n}\right) A_{n}^{\prime} U B \psi
$$

It should be remarked at this point in order to use Lemma 2 we have to reduce our problem to the case in which there exists a vector $\psi$ with the desired properties. We shall accomplish this making use of the assumption that $\pi$ has central support 1 .

Notice first that the center $\mathfrak{S}$ of $\mathfrak{A}^{-}$is left invariant by the automorphism $\tau$. In fact, if $B \in \mathfrak{E}, \tau(B) \in \mathfrak{A}^{-}$, and for every $A \in \mathcal{A}^{-}$,

$$
[\tau(B), A]=\tau\left[B, \tau^{-1}(A)\right]=0 .
$$

Therefore $\tau(B) \in \mathfrak{Q}^{\prime} \cap \mathfrak{A}^{-}$. q.e.d.

27 Commun. math. Phys., Vol. 2 
We now prove that the center is left pointwise invariant by the automorphism $\tau$, i.e., $\tau(B)=B$ for all $B \in \mathfrak{C}$. Consider the subalgebra $\mathfrak{A}_{\pi}$; since $\pi \in \mathfrak{A}^{-}$, its center is $\mathfrak{夭}_{\pi}[10]$. It is left pointwise invariant by $\tau^{\pi}$, since the latter is induced by a unitary operator in $\mathfrak{A}_{\pi}^{-}$.

Suppose now that $\mathbb{C}$ were not left pointwise invariant by $\tau$. There would exist then an element $B \in \mathfrak{C}$ such that $\tau(B) \neq B$. Without loss of generality, we can take $B$ (and therefore $\tau(B)$ ) self-adjoint. Since $\tau$ restricted to $\mathfrak{A}_{\pi}^{-}$coincides with $\tau^{\pi}$, we also have $\pi \tau(B)=\pi B$ i.e.

$$
\pi(\tau(B)-B)=0 \text {. }
$$

The operator $\tau(B)-B$ is self-adjoint. Let $P$ be any projection in its spectral family. From (20) one derives

$$
P \pi=0 \text {. }
$$

But then the projection $1-P$ belongs to the center of $\mathfrak{A}^{-}$and contains $\pi$ in the sense that $\pi(1-P)=\pi$. This contradicts the assumption that $\pi$ has central support 1 .

With $\xi$ an arbitrary vector in $\pi \mathscr{H}$, let $P_{\xi}$ be the projection onto the closure of $\mathfrak{2}_{\xi} . P$ is in the center of $\mathfrak{A}^{\prime}$ and is therefore invariant under $\tau$. Since $\pi$ has central support $I$, the projection $(1-P) \pi$ is non-zero. Let $\eta$ be a vector in $\left(1-P_{\xi}\right) \pi \mathscr{H}, P_{\eta}$ the projection onto the closure of $\mathfrak{R}_{\eta}$. $P_{\eta}$ belongs to the center and $P_{\xi} \cdot P_{\eta}=0$. Proceeding by induction, we construct a family of orthogonal central projections $P_{i}, i \in I$, of sum 1 . Each $P_{i}$ is left invariant by $\tau$ and therefore $\tau$ defines in a natural way an automorphism $\tau^{i}$ or each subalgebra $\mathfrak{A}_{i} \stackrel{\text { def }}{=} \mathfrak{A} P_{i}$. In $\pi P_{i} \mathscr{H}$ there exists by construction, a vector $\psi_{i}$ with the property $\mathfrak{R}_{\psi_{i}}=P_{i} \mathscr{H}$. In each subspace $P_{i} \mathscr{H}$ the conditions of Lemma 2 are verified and there exists therefore a family $V_{t}^{i}$ of unitary operators, $V_{t}^{i} \in P_{i} \mathfrak{A}^{-}$, such that $V_{t}^{i} A\left(V_{t}^{i}\right)^{-1}=\tau_{t}^{i}(A)$ for all $t$ and $A \in P_{i} \mathfrak{A}$.

Each $P_{i} H$ is left invariant by $\mathfrak{A}^{-}$and $\cup_{i} H_{i}=H$. We collect all this information in

Proposition 6. Under postulates $\alpha$ ) and $\beta$ ) there exists, for each $t$, a unitary operator $V_{t}$ such that $\tau_{t}(A)=V_{t} A V_{t}^{-1}$ for all $A \in \mathfrak{A}$. Moreover, $V_{t} \in \mathfrak{A}-$ for all $t$.

We want now to show that the $V_{t}^{\prime} s$ form a strongly continuous oneparameter group.

Let us first see that the $V_{t}^{\prime} s$ form a representation of the group of real numbers, i.e., $V_{t} \cdot V_{s}=V_{t+s}$. For all $\varphi \in \mathfrak{R}_{\psi}, \psi \in \mathscr{H}_{\pi}$,

$$
\begin{aligned}
V_{t+s} \varphi=\sum_{n} \tau_{t+s}\left(A_{n}\right) A_{n}^{\prime} U_{t+s} \psi & =\sum_{n} \tau_{t}\left(\tau_{s}\left(A_{n}\right)\right) A_{n}^{\prime} U_{s} U_{t} \psi \\
& =U_{t} U_{s} \cdot \varphi
\end{aligned}
$$

and the relation extends to $\mathscr{H}$ by continuity. q.e.d. In deriving (22) we 
have used the fact that the automorphisms $\tau_{t}$ form a representation of the group of real numbers and Eq. (19). (Notice that $U_{s} \in \mathfrak{A}_{\pi}^{-}, \forall s$ ).

We shall now proceed to prove strong continuity. It will in fact be sufficient to prove weak continuity as they are equivalent for groups of unitary operators.

We shall prove: for any $\eta, \varphi \in \mathscr{H}, \exists t_{0}$ such that

$$
|\langle\eta, V(t) \varphi\rangle-\langle\eta, \varphi\rangle|<1 \text { for } t<t_{0} .
$$

It will be sufficient to prove this inequality for $\varphi \in \mathfrak{R}_{\psi}, \psi \in \mathscr{H}_{\pi}$ when $\mathfrak{Q}_{\psi}$ is dense in $\mathscr{H}$.

For such $\varphi$, one has

$$
\langle\eta, V(t) \varphi\rangle=\sum_{n}\left\langle\eta, V(t) A_{n}^{\prime} A_{n} \psi\right\rangle=\sum_{n}\left\langle\eta, \tau_{t}\left(A_{n}\right) A_{n}^{\prime} U_{t} \psi\right\rangle .
$$

Now $\left\{U_{t}\right\}$ form a uniformly continuous group, therefore one can choose $t$ so small that

$$
\left|\sum_{n}\left\langle\eta, \tau_{t}\left(A_{n}\right) A_{n}^{\prime} U_{t} \psi\right\rangle-\sum_{n}\left\langle\eta, \tau_{t}\left(A_{n}\right) A_{n}^{\prime} \psi\right\rangle\right|<1 / 2 .
$$

(Notice that $\left\|\sum_{n} \tau_{t}\left(A_{n}\right) A_{n}^{\prime}\right\| \leqq \sum_{n}\left\|A_{n}\right\|\left\|A_{n}^{\prime}\right\|$ independent of $t$.) But $\tau_{t}$ is by assumption weakly continuous in $t$. Therefore, for $t$ sufficiently small

$$
\left|\sum_{n}\left\langle\eta, \tau_{t}\left(A_{n}\right) A_{n}^{\prime} \psi\right\rangle-\langle\eta, \varphi\rangle\right|<\mathbf{1} / 2
$$

Combining (25) and (26) we have finally that, for $t$ sufficiently small,

$$
|\langle\eta, V(t) \varphi\rangle-\langle\eta, \varphi\rangle|<1 \text {. q.e.d. }
$$

By STone's theorem there exists therefore a selfadjoint operator $H$ such that $V=e^{i H t}$. Since $V(t) \in \mathfrak{A}^{-}$for all $t$ and every bounded function of $H$ is in the von Neumann algebra generated by the $e^{i H t}, \forall t$, we conclude that all the elements of the spectral family of $H$ belong to $\mathfrak{A}^{-}$, i.e., $H \eta \mathfrak{A}^{-}$. It is easily checked that $H$ leaves $\mathscr{H}_{\pi}$ invariant and that its restriction to $\mathscr{H}_{\pi}$ coincides with $H_{\pi}$.

Also, $H$ is bounded below. We shall give the proof of this for the case in which there exists a vector $\psi \in \mathscr{H}_{\pi}$ such that $\mathfrak{T}_{\psi}$ is dense in $\mathscr{H}$. The general case is treated along the same lines modulo a suitable decomposition of the center.

The additive constant modulo which $H$ is defined can be chosen so that $H_{\pi}$ is positive and its spectrum starts at zero. Also, since $\langle\varphi, A(t) \psi\rangle$ has Fourier transform with support in $-M \leqq p \leqq M, 0 \leqq H_{\pi} \leqq M$.

We shall now show that $H$ is bounded below. We shall in fact prove that

if

$$
\int h(t)\langle\varphi, V(t) \chi\rangle d t=0
$$

$$
h \in \mathscr{L}_{\mathbf{1}} \cap \mathscr{L}_{2}, \int t|h(t)| d t<\infty \quad \text { and } \operatorname{supp} \tilde{h}(p) \subset\{p: p \leqq-2 M\},
$$
for all $\varphi, \chi \in \mathscr{H}$. 
Since

$$
\left|\int h(t) V(t) d t\right| \leqq|h|_{1}<\infty \text { and } V(t) \in \mathcal{A}
$$

it will suffice to prove it for states $\chi$ of the form $\chi=A \psi, A \in \mathfrak{A}$. Here $\psi$ is again a vector in $\pi \mathscr{H}$ such that $\mathscr{N}_{\psi}$ is dense in $\mathscr{H}$. By definition, $V(t) A \psi=A(t) U(t) \psi$ where

Therefore

$$
U(t)=\int_{0}^{M} e^{i \lambda t} d E(\lambda)
$$

$$
\begin{aligned}
& \int_{-\infty}^{\infty} h(t)\langle\varphi, V(t) \chi\rangle d t=\int_{-\infty}^{\infty} h(t) d t \int_{0}^{M} e^{i \lambda t} d t\langle\varphi, A(t) E(\lambda) \chi\rangle \\
= & \int_{-\infty}^{\infty} h(t) d t-\int_{0}^{M}(i t) e^{i \lambda t}\langle\varphi, A(t) E(\lambda) \chi\rangle+e^{i M t}\langle\varphi, A(t) \chi\rangle \\
= & -\int_{0}^{M} d \lambda \int t d t h(t)\langle\varphi, A(t) E(\lambda) \chi\rangle e^{i \lambda t}+\int_{-\infty}^{\infty} d t h(t)\langle\varphi, A(t) \chi\rangle e^{i M t}
\end{aligned}
$$

The interchange of order of integration is allowed since

$$
|\langle\varphi, A(t) E(\lambda) \chi\rangle| \leqq\|A\| \text { and } \int|h(t)| t d t<\infty
$$

by assumption. If $\tilde{h}(p)=0, p>-2 M$, then $\int e^{-i p t} t h(t) e^{i \lambda t}=0$, $p>-2 M+\lambda>-M$ and $\int e^{-i p t} h(t) e^{i M t} d t=0, p>-M$. Therefore, since $\chi \in \pi \mathscr{H}, \int h(t)<\varphi V(t) \chi>d t=0$. With $V(t)=\int e^{i \mu t} d F(\mu)$, this implies $d F_{\mu}=0, \mu<-2 M$, since the functions $\tilde{h}(p)$ with the properties specified above are dense in the space of continuous functions with support in $\{p: p<-2 M\}:$ We have therefore proved.

Theorem 2.18 Let $\mathfrak{A}$ be a $C^{*}$-algebra of operators on a separable Hilbert space $\mathscr{H}$. Let $\tau_{t}$ be a weakly continuous group of automorphisms of $\mathfrak{A}$ satisfying postulates $\alpha$ ) and $\beta$ ) stated above. Then there exists a strongly continuous group of unitary operators $V(t), V(t) \in \mathfrak{A}^{-}$, with infinitesimal generator $H, H \eta \mathfrak{A}^{-}$, such that for all $t$ and all $A \in \mathfrak{A}$, $\tau_{t}(A)=V(t) A V^{-1}(t)$.

In particular, if $\mathfrak{A}^{-}$represents the physical observables of a given theory and $\tau_{t}$ is, e.g., the group of time translations, then Theorem 2 asserts that the Hamiltonian exists and that the energy is a physical observable.

We shall conclude with a corollary which has an obvious application, e.g., to the measurability of momentum in relativistic theories.

Corollary. Let the algebra $\mathfrak{A}$ have a weakly continuous $n$-parameter Abelian group of automorphisms $\tau_{t_{1}, \ldots, t_{n}}$ and let postulates $\alpha$ ) and $\beta$ ) be satisfied for $n$ one-dimensional subgroups $\tau_{\alpha_{11} t, \ldots, \alpha_{1 n} t}, \ldots, \tau_{\alpha_{n 1} t, \ldots, \alpha_{n n} t}$, such that the matrix $\alpha_{i j}$ is invertible. Then such automorphisms are

${ }_{18}$ A proof that, if the strongly continuous group $V(t)$ exists and satisfies assumption $\alpha$ ) (assumption $\beta$ is automatically satisfied) its generator is affiliated to $\mathfrak{A}^{-}$, has been given by a different method by H. J. Borchens [11]. 
implemented by a strongly continuous $n$-parameter Abelian group of unitary operators and all the generators of one-dimensional subgroups are affiliated to $\mathfrak{A}-$.

Acknowledgments. The author would like to thank R. KADIson for having communicated to him the results of [2] prior to publication and for many enlightening discussions. The author would also like to thank $\mathrm{H}$. BorCHERs for useful discussions and M. L. Motchane for his warm hospitality at the I.H.E.S. The work was completed while the author was at the Courant Institute of Mathematical Sciences, New York University, under a G. Sloan Foundation Grant.

\section{Appendix 1}

Let $I_{0}$ be a denumerable set of points on the real $t$-axis. We want to prove

Lemma A1. If $B \in \mathfrak{M}$, for every pair $\varphi, \psi \in \mathscr{H}_{\pi}$ (not necessarily normalized to 1) there exists a sequence $A_{1}, A_{2}, \ldots, A_{n} \in \mathfrak{Q}_{\pi}$, such that on $I_{0}$,

$$
\langle\varphi, A(t) \psi\rangle \underset{n \rightarrow \infty}{\longrightarrow}\langle\varphi, B(t) \psi\rangle \text {. }
$$

For $\psi \in \mathscr{H}_{\pi}, t \in I$, let $\sigma_{t}$ be the normal state of $\mathfrak{A}_{\pi}$ defined by ${ }^{19}$

$$
\sigma_{t}(A)=\langle\psi, A(t) \psi\rangle, \quad A \in \mathfrak{A}_{\pi} .
$$

Here and in what follows $A=\left.A(t)\right|_{t=0}$.

Since $\sigma_{t}$ is a normal state, there exists a denumerable orthogonal set of vectors $\psi_{n}^{t} \in \mathscr{H}_{\pi}, \sum_{n}\left|\psi_{n}^{t}\right|=M<\infty\left(M=\sigma_{t}(I)=\right.$ indep. of $\left.t\right)$ such that

for all $A \in \mathfrak{Q}_{\pi}$.

$$
\sigma_{t}(A)=\sum_{n}\left\langle\psi_{n}^{t}, A \psi_{n}^{t}\right\rangle
$$

We shall denote by $\mathscr{H}_{t}$ the (separable) Hilbert space spanned by the vectors $\psi_{n}^{t}, n=1,2 \ldots$ and by $\mathscr{H}_{I_{0}}$ the (separable) Hilbert space spanned by the vectors in $\bigcup_{t \in I_{0}} \mathscr{H}_{t}$. One has $\mathscr{H}_{I_{0}} \subset \mathscr{H}_{\pi}$.

Let $E$ be the projection from $\mathscr{H}$ to $\mathscr{H}_{I_{0}}$; it satisfies $E \pi=E$. Let $B$ be an element in $\mathfrak{M}$. Since $\mathfrak{M} \subset \mathfrak{A}_{\pi}^{-} \subset \mathfrak{A}^{-}, B$ is in the strong closure of $\mathfrak{A}$. Without loss of generality, we shall take $\|B\|=1$. By KaPLANsky's Density Theorem [10] (p. 96, Th. 3) $B$ is in the strong closure of $\mathfrak{A}_{1}$. Since $\|E A E\| \leqq\|A\|$ for all bounded $A, E B E$ is in the strong closure of $E \mathfrak{A}_{1} E$. The set $E \mathfrak{A}_{1} E$ is bounded and, by construction, $E \mathfrak{A} E \subset \mathscr{L}\left(\mathscr{H}_{I_{0}}\right)^{20}$. Since $\mathscr{H}_{I_{0}}$ is separable, $\mathscr{L}\left(\mathscr{H}_{I_{0}}\right)$ is countably decomposable. There exists therefore [10] (p. 33, Cor.) a sequence of elements in $E \mathfrak{A} E$ which converges strongly to $E B E$.

10 We have already remarked that Postulate $\beta$ guarantees that $\sigma_{t}$ is a normal state for every $\psi \in \mathscr{H}$ and real $t$.

20 $\mathscr{L}(\mathscr{H})$ is the (VoN NEUMANN) algebra of all bounded operators on the Hilbert space $\mathscr{H}$. 
Let $A_{1}, A_{2}, \ldots$ be a sequence in $\mathfrak{A}$ such that $E A_{n} E \underset{n \rightarrow \infty}{\longrightarrow} E B E$, where the limit is taken in the strong sense.

Consider for $\psi, \varphi \in \mathscr{H}_{\pi}$ the sequence of functions $\left\langle\psi, A_{n}(t) \psi\right\rangle$. Since $E \pi=E$, this is a sequence of entire functions of exponential type $M$. Let $t_{0} \in I_{0}$. Then

$$
\left\langle\psi,\left(A_{n}\left(t_{0}\right)-B\left(t_{0}\right)\right) \psi\right\rangle=\sum_{k=1}^{\infty}\left\langle\psi_{k}^{t_{0}},\left(A_{n}-B\right) \psi_{k}^{t_{0}}\right\rangle .
$$

There exists an integer $k_{0}$ such that $\sum_{k>k_{0}}\left|\psi_{k}^{t_{0}}\right|^{2} \leqq 1 / 4$. Also, there exists an integer $n_{0}$ such that, for $n>n_{0},\left|\left\langle\psi_{k}^{t_{0}},\left(A_{n}-B\right) \psi_{k}^{t_{0}}\right\rangle\right|<1 / 2 k_{0}$, since $E A_{n} E \rightarrow E B E$ and $E \psi_{k}^{t_{0}}=\psi_{k}^{t_{0}}, \forall k$. Therefore there exists an integer $n_{0}$ such that

$$
\left|\left\langle\psi,\left(A_{n}\left(t_{0}\right)-B\left(t_{0}\right)\right) \psi\right\rangle\right|<1 \text { for } n>n_{0} .
$$

This shows that for every $\psi \in \mathscr{H}_{\pi}$ the sequence of (entire, of exponential type $M)$ functions $\left\langle\psi, A_{n}(t) \psi\right\rangle$ converges to the function $\langle\psi, B(t) \psi\rangle$ on the denumerable set $I_{0}$. By polarization, one has the same results for the sequence of functions $\left\langle\varphi, A_{n}(t) \psi\right\rangle, \varphi, \psi \in \mathscr{H}_{\pi}$.

\section{Appendix 2}

Lemma A 2. Let $f_{1}(z), f_{2}(z), \ldots$ be a sequence of entire functions of exponential type 1 , converging pointwise on the real axis to the continuous function $f(t)$, uniformly on the compact sets.

Let $\left|f_{n}(t)\right| \leqq 1,|f(t)| \leqq 1$, for all integers $n$ and real $t$. Then $f(t)$ is the restriction to the real axis of an entire function of exponential type 1 .

Let $\gamma(z)$ be entire of exponential type $\sigma$, square integrable and bounded by 1 on the real $t$-axis.

Define

$$
g_{n}(z) \stackrel{\text { def }}{\equiv} f_{n}(z) \gamma(z) ; g(t) \stackrel{\text { def }}{\equiv} f(t) \gamma(t), \quad t \text { real . }
$$

For all $n, g_{n}(z)$ is entire of exponential type $1+\sigma$ and its restriction to the real $t$-axis is square-integrable. Also, $g(t) \in \mathscr{L}_{2}$ and is continuous. Given $\varepsilon>0$, let $k$ be a compact set such that

One has then

$$
\int_{k^{+}}|\gamma(t)|^{2} d t<\varepsilon / 4
$$

$$
\begin{aligned}
\left\|g_{n}-g\right\|_{2}= & \int\left|f_{n}(t)-f(t)\right|^{2}|\gamma(t)|^{2} d t \\
& \leqq \int_{k}\left|f_{n}(t)-f(t)\right|^{2} d t+\int_{k^{\perp}}|\gamma(t)|^{2} d t
\end{aligned}
$$

Due to uniform convergence on the compact sets, there exists an integer $n_{0}$ such that, for $n>n_{0}, \int_{k}\left|f_{n}(t)-f(t)\right|^{2} d t<1 / 2$. Therefore, for $n>n_{0},\left\|g_{n}-g\right\|_{2}<\varepsilon$. 
The sequence $g_{1}, g_{2}, \ldots$ converges in $\mathscr{L}_{2}$ to $g \in \mathscr{L}_{2}$ and this implies convergence of the Fourier transforms $\tilde{g}_{1}, \tilde{g}_{2}, \ldots$ to $\tilde{g}$.

By assumption, the support of $\tilde{g}_{n}$ is contained in $\{p:|p| \leqq 1+\sigma\}$. This implies that $\tilde{g}$ has also support in $\{p:|p| \leqq 1+\sigma\}$. Since this holds true for every choice of the entire function $\gamma$ of exponential type $\sigma$, one has $\operatorname{supp} f \subset\{p:|p| \leqq 1+2 \sigma\}$. Lemma 2 follows now from the arbitrariness of $\sigma>0$.

Corollary. Let $f_{1}(z), f_{2}(z), \ldots$ a sequence of entire functions of exponential type 1 , bounded by 1 on the real axis and converging on a dense subset $I_{0}$ of the real axis to an entire function $f(z)$ of exponential type $\alpha$. Then $\alpha=1$.

We must prove uniform convergence on the compact sets, and then apply Lemma A2. Let $K$ be compact, $P_{\delta}$ a partition of $K$ in $m_{0}$ intervals $K_{i}$ of length $\delta, i=1,2, \ldots$.

Let $t_{i}$ be any point in $K_{i} \cap I_{0}$ (since $I_{0}$ is dense in $K$, the set $K_{i} \cap I_{0}$ is not empty). Writing $f\left(t_{i}\right)$ for $\left.f(t)\right|_{t=t_{i}}$, for all $n$ and real $t$,

$$
\left|f_{n}(t)-f(t)\right| \leqq\left|f_{n}\left(t_{i}\right)-f\left(t_{i}\right)\right|+2 \alpha \delta \quad \text { for some } t_{i} .
$$

Given $\varepsilon>0$, take $\delta<\varepsilon / 4 \alpha$ and let $n_{i}$ be such that $\left|f_{n}\left(t_{i}\right)-f\left(t_{i}\right)\right|<$ $<\varepsilon / 2$ for $n>n_{i}$; let $n_{0} \stackrel{\text { def }}{=} \sup _{i=1, \ldots, m_{0}} n_{i}$. Then, for $n>n_{0}$,

$$
\left|f_{n}(t)-f(t)\right| \leqq \varepsilon / 2+\varepsilon / 2=\varepsilon \quad \text { uniformly in } K \text {. q.e.d. }
$$

\section{References}

[1] Araki, H.: Progr. Theoret. Phys. 32, 844 (1964).

[2] Kadison, R.: Ann. Math. (to appear).

[3] Doplicher, S.: Commun. Math. Phys. 1, 1-5 (1965).

[4] Kadison, R.: Ann. Math. 66, 304 (1957).

[5] Schwartz, L.: Theorie des distributions. Paris: Hermann 1959.

[6] Riesz, F., et B. Sz.-Nagy: Leçons d'Analyse Fonctionelle, Academie des Sciences d'Hongrie, 1952.

[7] Sakar, S.: The theory of $W^{*}$-algebras. Yale University Lectures Notes, 1962.

[8] Boas, R.: Entire functions. New York: Academic Press 1964.

[9] Segal, I.: Duke Math. J. 18, 221 (1951).

[10] Dixmier, J.: Les algébres d'operateurs dans l'espace Hilbertien. Paris: Gauthier-Villars 1957.

[11] Borchers, H. J.: Commun. Math. Phys. 2, 49—54 (1966). 
